\title{
The role of the NLRP3 inflammasome in gout
}

\author{
This article was published in the following Dove Press journal: \\ Journal of Inflammation Research \\ 12 March 2011 \\ Number of times this article has been viewed
}

\author{
Sarah R Kingsbury ${ }^{1,2}$ \\ Philip G Conaghan ${ }^{1,2}$ \\ Michael F McDermott ${ }^{1,2}$ \\ 'Section of Musculoskeletal Disease, \\ Leeds Institute of Molecular Medicine \\ ${ }^{2} \mathrm{NIHR}$ Leeds Musculoskeletal \\ Biomedical Research Unit, Leeds \\ Institute of Molecular Medicine, \\ University of Leeds, Leeds, UK
}

\begin{abstract}
Gout is an inflammatory arthritis characterized by abrupt self-limiting attacks of inflammation caused by precipitation of monosodium urate crystals (MSU) in the joint. Recent studies suggest that orchestration of the MSU-induced inflammatory response is dependent on the proinflammatory cytokine IL-1 $\beta$, underlined by promising results in early IL- 1 inhibitor trials in gout patients. This IL-1-dependent innate inflammatory phenotype, which is observed in a number of diseases in addition to gout, is now understood to rely on the formation of the macromolecular NLRP3 inflammasome complex in response to the MSU 'danger signal'. This review focuses on our current understanding of the NLRP3 inflammasome and its critical role in MSU-crystal induced inflammatory gout attacks. It also discusses the management of treatment-resistant acute and chronic tophaceous gout with IL-1 inhibitors; early clinical studies of rilonacept (IL-1 Trap), canakinumab (monoclonal anti-IL-1 $\beta$ antibody), and anakinra have all demonstrated treatment efficacy in such patients.
\end{abstract}

Keywords: gout, inflammasome, NLRP3, IL-1

\section{Introduction}

Gout is one of the oldest recognized afflictions in humans, with a documented history reaching back to the Egyptians in 2640 BC. Described by Hippocrates as the 'arthritis of the rich' due to its association with food and alcohol, gout is an inflammatory arthritis caused by precipitation of monosodium urate (MSU) in articular joints and bursal tissues of individuals with hyperuricemia and is characterized by abrupt, self-limiting attacks of joint inflammation. Although MSU was identified in gout in the $1700 \mathrm{~s}^{1}$ and shown to be the causative agent in $1899,{ }^{2}$ the mechanisms by which MSU crystals trigger acute inflammation have only recently begun to be understood.

Proinflammatory cytokines undoubtedly have a critical role in orchestrating the inflammatory reaction to MSU crystals. Recent studies have implicated interleukin-1 $\beta$ (IL-1 $\beta)$ as a key regulatory proinflammatory cytokine in gout, promoting a neutrophil influx into the synovium and joint fluid that is the pathological hallmark of an acute inflammatory attack. ${ }^{3}$ Compelling evidence for IL- $1 \beta$ 's role in gout-associated pain and inflammation is provided by studies in both animals and man. In a murine gout model, inflammation following MSU injection into the mouse ankle joint was significantly reduced both in mice deficient for the IL-1 receptor and in wildtype mice treated with the IL-1 inhibitor IL1 Trap (rilonacept). ${ }^{4,5}$ In clinical studies, a rapid response was observed in patients with acute gouty arthritis following treatment with a number of anti-IL-1 agents (IL-1RA [anakinra], IL-1Trap, and anti-IL-1 $\beta$ monoclonal antibody [canakinumab]) thereby reinforcing the argument for a pivotal
Correspondence: Michael F McDermott NIHR-Leeds Musculoskeletal Biomedical Research Unit (NIHR-LMBRU), Level 6, Room 6.19a, Leeds Institute of Molecular Medicine, Wellcome Trust Brenner Building, St James's University Hospital, Leeds LS9 7TF, UK

Tel +44 II3343864I

Email m.mcdermott@leeds.ac.uk 
role for IL-1 $\beta$ in gout pathogenesis. ${ }^{6-8}$ It is currently unclear whether IL- $1 \alpha$ is also involved in gout pathogenesis since early IL-1 inhibitors (anakinra and rilonacept) inhibit both members of the IL-1 family; however the rapid and sustained response of gout patients to canakinumab in a recent Phase II study $^{9}$ suggests that IL- $1 \beta$ may be the more essential of the two IL-1 cytokines.

IL- $1 \beta$ is produced as an inactive pro-molecule by immune cells, including macrophages, monocytes, and dendritic cells, following immune stimulation and is then cleaved to its active, p17 form by caspase-1 (also termed IL-1-converting enzyme or ICE), itself existing as a proform until the initiation of the innate immune response. The mechanism of caspase- 1 and IL- $1 \beta$ activation following MSU deposition has been the focus of a number of recent high profile studies and is now understood to involve the formation of macromolecular complexes termed 'inflammasomes'. Since its discovery less than a decade ago, the NLRP3 inflammasome complex has been implicated as a key regulator of the innate inflammatory phenotype of several diseases, including gout and type 2 diabetes. In addition, mutations in NLRP3 inflammasome components have been shown to be responsible for the dysregulated IL-1 $\beta$ production observed in a number of (auto) inflammatory disorders, including Muckle-Wells syndrome (MWS), familial cold autoinflammatory syndrome (FCAS), and chronic infantile neurologic, cutaneous, and articular syndrome/neonatal onset multisystem inflammatory disease (CINCA/NOMID).

In this review we discuss the mechanisms and pathophysiology of the inflammasome complex and, in particular, its role in mediating the innate immune inflammatory response to MSU crystal deposition in gout.

\section{Structure and molecular mechanisms involved in inflammasome activation}

The innate immune system is characterized by its ability to recognize and respond to an array of pathogens (including viruses, bacteria, and fungi) and to endogenous molecules released by host cells as a result of necrosis, infections, membrane, ${ }^{10}$ and lysosomal ${ }^{11,12}$ damage, injury, or certain pathological conditions (including mammalian dsDNA, extracellular ATP, and MSU crystals), ${ }^{13-16}$ through detection of pathogen-associated and danger-associated molecular patterns (PAMPS and DAMPs respectively). This response is mediated by germline-encoded receptors termed patternrecognition receptors (PRRs).
Among the PRR families that have been described to date include the Toll-like receptors (TLRs), which are associated with extracellular (TLRs 1, 2, 5, 6, and 10) or endosomal (TLRs 3, 7, 8, and 9) membranes and the intracellular RIG-like helicases (RLHs) and NOD-like receptors (NLRs). TLRs, of which there are 10 known in humans (TLR 1-10) and 12 in mice (TLR 1-9 and 11-13), are type 1 transmembrane proteins composed of three major domains and characterized by leucine-rich repeats (LRRs) in the ectodomain which mediate PAMP/DAMP recognition. TLRs are expressed on many cell types and are known to respond to a variety of PAMPs and DAMPs, including bacterial DNA, ${ }^{13}$ lipopolysaccharide (LPS), ${ }^{16}$ peptidoglycan, ${ }^{17}$ teichoic acids, ${ }^{17}$ flagellin ${ }^{18}$ pilin, ${ }^{19,20}$ viral dsRNA, ${ }^{21}$ and fungal zymosan. ${ }^{22}$ Upon activation they differentially trigger signaling cascades that mediate production of the transcription factors NF- $\kappa \beta$, AP- 1 , and interferon-regulatory factor (IRF)-3, which, in turn, mediate transcription of proinflammatory cytokines, such as interleukins, interferons, and TNF, that drive the inflammatory response. The TLRs have been the subject of extensive investigation and review over recent years and will therefore not be discussed in depth in this review. The two known RLHs, RIG-1 and MDA5, are viral RNA sensors which, upon stimulation, activate NF- $\mathrm{KB}$ and IRF3/7 leading to transcription of type I interferons. ${ }^{23}$

The intracellular NLRs, like TLRs, are capable of recognizing both PAMPs and DAMPs, and have become a focal point for investigation of the immune response to foreign agonists and endogenous molecules. Known activators of the NLRs include bacterial muramyl dipeptide (MDP), ${ }^{24}$ microbial toxins (such as alpha hemolysin of Staphylococcus aureus), ${ }^{25}$ RNA of bacterial and viral origin, ${ }^{26,27} \mathrm{MSU}$ and calcium pyrophosphate dihydrate (CPPD) crystals, ${ }^{15}$ alum, ${ }^{28}$ asbestos, ${ }^{29,30}$ silica, ${ }^{29,30}$ and extracellular ATP ${ }^{14}$ (Table 1). To date, at least 23 human and 34 murine NLR genes have been identified, although the physiological functions of many of these are still poorly understood. ${ }^{31}$ NLRs are characterized by three domains: a C-terminal LRR 'sensor' domain, a central nucleotide-binding domain (NOD, also called NACHT domain) which regulates self-oligomerization and an N-terminal interaction domain which mediates proteinprotein interactions with downstream signaling intermediates (Figure 1). The N-terminal domain can also be used to categorize the NLRs into 5 subfamilies: NLRA (containing an acidic transactivation domain), NLRB (containing a baculovirus inhibitor of apoptosis protein repeat [BIR]), NLRC (containing a caspase-recruitment domain [CARD]), 
Table I Inflammasome activators

\begin{tabular}{|c|c|c|c|}
\hline \multirow[t]{2}{*}{ Inflammasome } & \multicolumn{3}{|l|}{ Stimulus } \\
\hline & Whole pathogen & PAMP & DAMP \\
\hline \multirow[t]{2}{*}{ NLRPI } & & MDP & \\
\hline & & Bacillus anthracis lethal toxin & \\
\hline \multirow[t]{16}{*}{ NLRP3 } & Staphylococcus aureus & DNA & Uric acid \\
\hline & Listeria monocytogenes & R837 & Cholesterol \\
\hline & Neisseria gonorrhoea & MDP & Asbestos \\
\hline & Escherichia coli & LPS & Silica \\
\hline & Mycobacterium marinum & $\alpha$-toxin & Nanoparticles \\
\hline & Candida albicans Influenza A & Bacterial RNA & $\beta$ amyloid \\
\hline & Adenovirus & Poly(I:C) & Hemozoin crystals \\
\hline & Sendai virus & Nigericin & Calcium pyrophosphate \\
\hline & Encephalomyocarditis & Listeriolysin $O$ & dihydrate crystals \\
\hline & Vaccinia virus & Aerolysin & Alum \\
\hline & & Maitotoxin & Islet amyloid polypeptide \\
\hline & & & UVB \\
\hline & & & Mutations \\
\hline & & & ATP \\
\hline & & & Hyaluron \\
\hline & & & Glucose \\
\hline \multirow[t]{6}{*}{ NLRP4 (IPAF) } & Salmonella typhimurium & Flagellin & \\
\hline & Shigella flexneri & & \\
\hline & Legionella pneumophilia & & \\
\hline & Pseudomonas aeruginosa & & \\
\hline & Vaccinia virus & & \\
\hline & Mouse cytomegalovirus & & \\
\hline AIM2 & & & dsDNA \\
\hline
\end{tabular}

Abbreviations: DAMP, danger-associated molecular pattern; LPS, lipopolysaccharide; MDP, muramyl dipeptide; PAMP, pathogen-associated molecular pattern; poly(I:C), polycytidylic acid.

NLRP (containing a Pyrin domain), and NLRX (containing an unknown domain). Of these the best characterized, and the most important in the context of this review, are the NLRPs and, in particular, NLRP3.

NLRs respond to PAMPs/DAMPs through the formation of 'inflammasomes'; multimeric cytoplasmic protein complexes which act as molecular platforms for the activation of inflammatory caspases following stimulation by foreign agonists. ${ }^{32}$ A typical inflammasome is composed of an NLR, an adaptor protein such as apoptosis-associated speck-like protein containing a CARD (ASC) and an effector caspase that activates proinflammatory cytokines, in particular IL-1 $\beta^{32}$ (Figure 1). To date three NLR proteins have been identified to form inflammasomes: NLRP1, NLRP3, and NLRC4 (also known as IPAF). NLRP3 (NALP3, also known as cryopyrin or PYPAF1 [Pyrin-containing Apaf1like protein 1]), probably the best understood NLRP, has been shown to be involved in the recognition of numerous exogenous and host ligands, including bacterial RNA, ATP and microbial toxins. ${ }^{33}$ Stimulation of the NLRP3 LRR domain by a foreign agonist is postulated to unfold the NLRP3 molecule enabling recruitment of the ASC adaptor proteins and pro-caspase-1. This process of inflammasome assembly results in cleavage of pro-caspase- 1 to produce active caspase- 1 , which in turn cleaves pro-IL-1 $\beta$ to produce IL-1 $\beta$ and also activates IL-18.

Notably no study has yet demonstrated direct interaction between the LRRs of NLRs and their respective activators, suggesting that indirect mechanisms of sensing and activation may be involved. Conceptually, given the diverse array of molecules now known to activate NLRs it follows that additional indirect mechanisms may play a role in mediating inflammasome activation. Although the precise molecular details have not yet been fully elucidated, current evidence points to the potential roles of three signaling pathways in mediating agonist recognition and inflammasome activation. Firstly, recent studies suggest a central role for P2X7 receptor-mediated $\mathrm{K}+$ efflux in NLRP3 activation, with prevention of $\mathrm{K}+$ efflux abolishing NLRP3 in response to almost all known activators. ${ }^{34-36}$ Secondly, activation of the NLRP3 inflammasome by crystals and particulates appears to involve the phagocytic pathway, with particle uptake disrupting the phagolysosome acidic compartment and subsequent release of capthepsin B. ${ }^{12,37,38}$ Finally, reactive oxygen 


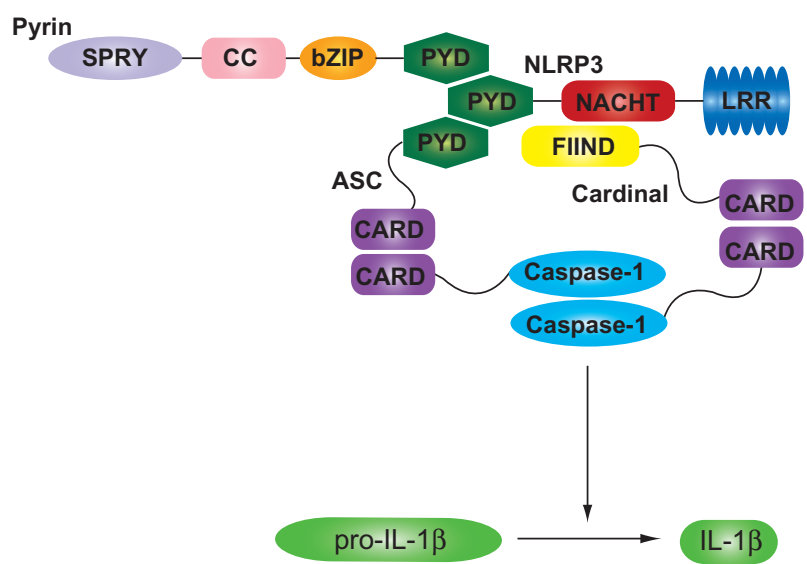

Figure I The NLRP3 inflammasome complex. The NLRP3 inflammasome complex is formed through homotypic interactions between the CARD and PYD domains of NLRP3, Pyrin, and ASC. An additional adaptor protein Cardinal is also required to facilitate activation of procaspase-I, which in turn cleaves prolL-I $\beta$ to IL-I $\beta$.

Abbreviations: ASC, apoptosis-associated speck-like protein; bZIP, basic leucine zipper domain; CARD, caspase activation and recruitment; CC, coiled-coil domain; FIIND, domain with a function to find; IL, interleukin; LRR, leucine-rich repeat; NACHT, domain present in neuronal apoptosis inhibitor protein (NAIP) major histocompatibility complex class II transactivator; NLRP3, NACHT domain, LRR domain, and Pyrin domain-containing protein; PYD, Pyrin domain; SPRY, SPRY domain.

species (ROS) generation has been suggested to be critical for inflammasome activation in response to a number of stimuli. Inhibition of ROS generation, either through pharmacological inhibitors of NAPDH oxidase or siRNA-mediated knockdown, prevents IL- $1 \beta$ release in response to MSU and a number of other stimuli. ${ }^{29,30}$ However, the role of ROS is still unclear since further studies suggest that increased ROS production may actually inhibit caspase- 1 activation. ${ }^{39}$ It is conceivable that the mechanisms involved in inflammasomeactivation may differ according to both agonist and cell type. A recent study supports this hypothesis, demonstrating that whilst IL-1 $\beta$ release by in vitro monocyte cultures required only a single exogenous stimulus, two distinct exogenous stimuli were required for IL- $1 \beta$ production in macrophage cultures. ${ }^{40}$ The difference between these two cell types is understood to be due to the presence of constitutively active caspase- 1 in monocytes and also the ability of monocytes to release endogenous ATP, which can act as a second stimulus to trigger IL-1 $\beta$ release. In contrast, macrophages require a primary signal to induce transcription and translation and a second distinct signal for caspase activation, which leads to IL-1 $\beta$ processing and release.

Recently an additional inflammasome sensor was described, which triggers caspase- 1 and IL- $1 \beta$ cleavage in an ASCdependent, NLR-independent manner. The AIM2 inflammasome contains a HIN200 domain that binds cytosolic DNA, including that of bacterial, viral, and mammalian origin, leading to recruitment of the ASC adaptor protein to its PYD and subsequent activation of caspase- 1 and IL-1 $\beta^{41-44}$

Negative regulation of inflammasomes is mediated by a family of small proteins composed of a CARD or PYD only domain. Five CARD-only proteins have been identified to date - ICEBERG, Pseudo-ICE, Caspase-12, INCA (Inhibitory CARD), and Nod2-S - which, with the exception of the Rip2-interacting Nod2-S protein, regulate NLRP3 activity through interaction with the caspase-1 CARD domain, thereby preventing its interaction with other inflammasome components. ${ }^{45-48}$ Two PYD-only proteins, POP1 and POP2, interact with ASC or NLRP thereby preventing their recruitment into inflammasome complexes. Limited data currently exist as to how these negative regulators are themselves regulated, their different roles in mediating inflammasome response and their involvement in inflammatory disease. Notably, Caspase-12 deficient mice show increased IL-1 $\beta$ secretion $^{49}$ whilst caspase-12 gene polymorphisms have been linked to the modulation of inflammatory and innate immune responsiveness. ${ }^{50}$ In addition to these molecular mechanisms of regulation, CD4+ effector and memory T-cells were shown to negatively regulate inflammasome activity in a CD40L-dependent manner. ${ }^{51}$ Inhibition of the NLRP3 inflammasome was found to be dependent on both T-cell activation and antigenic stimulation, suggesting that migration of T-lymphocytes to inflammatory sites may provide a feedback loop to regulate the production of inflammatory mediators during the later phases of inflammatory responses.

\section{Pathophysiology and role in various disease states}

Inflammasomes are now understood to have a fundamental role in the development of autoinflammatory diseases and additional roles in infection control, development of immune pathologies, and recognition of tissue damage. The last decade has seen the emergence of evidence supporting this concept, with the first established link to pathological processes provided by genetic studies of autoinflammatory diseases. Cryopyrinopathies (also referred to as hereditary periodic fever syndromes or cyropyrin-associated periodic syndromes) form a clinical continuum of autoinflammatory disorders characterized by recurrent fevers and inflammation. Most cases of these three disorders (FCAS, MWS, and NOMID in order of increasing severity) are now understood to be caused by autosomal dominant or de novo gain-of-function mutations in NLRP3. Over 40 mutations have been identified in NLRP3 ${ }^{52-56}$ 
each producing a constitutively active form of NLRP3, most likely due to spontaneous NLRP3 oligomerization, and hence elevated levels of secreted IL-1 $\beta$, which drive the inflammation. In keeping with this, gene-targeted mice harboring mutations equivalent to those found in MWS exhibit hyperactive NLRP3 inflammasomes leading to elevated IL-1 $\beta .^{57}$ This discovery has revolutionized the clinical management of these diseases, with patients responding well to IL-1 $\beta$ antagonism, for example by anakinra, ${ }^{58-60}$ next-generation IL-1 $\beta$ antagonists ${ }^{61-65}$ or caspase-1 inhibitors. ${ }^{66}$

Two additional autoinflammatory diseases, familial Mediterranean fever (FMF) and pyogenic arthritis, pyodermagangrenosum, and acne (PAPA) are also caused by mutations in the inflammasome pathway; FMF by mutations in the Pyrin-encoding MEFV gene ${ }^{67}$ and the syndrome of PAPA by mutations in the gene encoding proline-serine-threoninephosphatase-interacting protein (PSTPIP1), which binds Pyrin through its Src-homology-3 domain. ${ }^{68,69}$ Genetic variants in NLRP3 and other inflammasome-related genes have also been related to susceptibility and disease severity in a number of other chronic inflammatory disorders, including rheumatoid arthritis, ${ }^{70,71}$ Crohn's disease, ${ }^{72}$ gout, ${ }^{73}$ and the recently identified deficiency of the interleukin-1-receptor antagonist (DIRA). ${ }^{74}$

In addition to causal mutations in the NLRP3 inflammasome pathway, the pathology of a number of inflammatory diseases has been linked to the activation of this inflammasome by disease-specific inflammasome agonists. For example, the fibrillar peptide amyloid- $\beta$, which accumulates to form the senile plaques responsible for the pathogenesis of Alzheimer's disease, ${ }^{37}$ is now known to be an inflammasome activator. Studies suggest that NLRP3, caspase-1, and IL-1 $\beta$ are essential for the recruitment of microglia to exogenous amyloid- $\beta$ in the brain and for microglial synthesis of proinflammatory and neurotoxic factors, indicating a critical role for the inflammasome pathway in the innate immune response to amyloid- $\beta$ and the subsequent tissue damage that is the hallmark of Alzheimer's disease. In type 2 diabetes, islet amyloid polypeptide, which forms amyloid deposits in the pancreas, activates NLRP3, resulting in IL-1 $\beta$ release and inducing apoptosis of pancreatic beta cells ${ }^{75}$ whilst cholesterol crystals have been shown to trigger inflammasome activation in atherosclerosis. ${ }^{76,77}$ Late-stage human melanoma cells exhibit autoinflammatory characteristics, spontaneously secreting active IL-1 $\beta$ due to constitutive assembly and activation of NLRP $3 .{ }^{78} \mathrm{IL}-1$ mediated autoinflammation drives macrophage chemotaxis and angiogenesis, thereby directly contributing to disease development and progression. ${ }^{78}$ The inflammasome has also been linked to the progression of chronic kidney disease ${ }^{79}$ and to a number of viral infections. ${ }^{80-82}$ As the latter have been reviewed in depth recently, ${ }^{83}$ they will not be discussed further here. Although the above examples point clearly to a role for NLRP3 inflammasome activation in an array of human diseases, the involvement of this pathway in various disease processes is far from straightforward, as most notably highlighted by a recent paper demonstrating that in colitis-associated cancer the NLRP3 inflammasome plays a protective role, with ASC-/- and caspase-1-/- mice showing increased disease severity and morbidity. ${ }^{84}$

\section{The role of the NLRP3 inflammasome in acute and chronic gout}

Although the causal agent of gout has been understood for more than a century, the mechanisms underlying MSU crystal-induced inflammation have only recently begun to be unraveled. The pathological hallmark of a gout attack is considerable neutrophil influx into the synovium and joint fluid, ${ }^{3}$ however since neutrophils are absent in the normal joint the primary event following precipitation of MSU within the joint is believed to be the interaction of MSU crystals with resident joint cells, principally the synovial lining cells, which in turn trigger neutrophil ingress. Recent in vitro studies implicate mononuclear phagocytes as playing a central role in the initial response to MSU precipitation. The exposure of monocyte cell lines to MSU crystals leads to the production of proinflammatory cytokines, in particular $\mathrm{IL}-1 \beta,{ }^{85,86}$ and it is now understood that phagocytosis of MSU crystals is central to this process ${ }^{12}$ (Figure 2). Following the recognition that MSU crystals may act as a 'danger' signal to cells in a similar way to microbial pathogens, ${ }^{87}$ Martinon et al demonstrated the NLRP3 inflammasome to be critical in sensing MSU deposition and subsequent activation of the downstream innate immune response. ${ }^{15}$ Macrophages from mice deficient in various components of the inflammasome, including caspase-1, ASC, and NLRP3, were unable to activate IL-1 $\beta$ in response to MSU stimulation. Notably, these mice also showed impaired neutrophil influx following intraperitoneal MSU injection, demonstrating the NLRP3 inflammasome as a critical link between the well-established causal stimulus of gout and the subsequent pathological hallmark of an acute gout attack. Similar results were also demonstrated with CPPD crystals, the causal agent of pseudogout, suggesting that similar mechanisms are involved in regulating the inflammatory response in both 


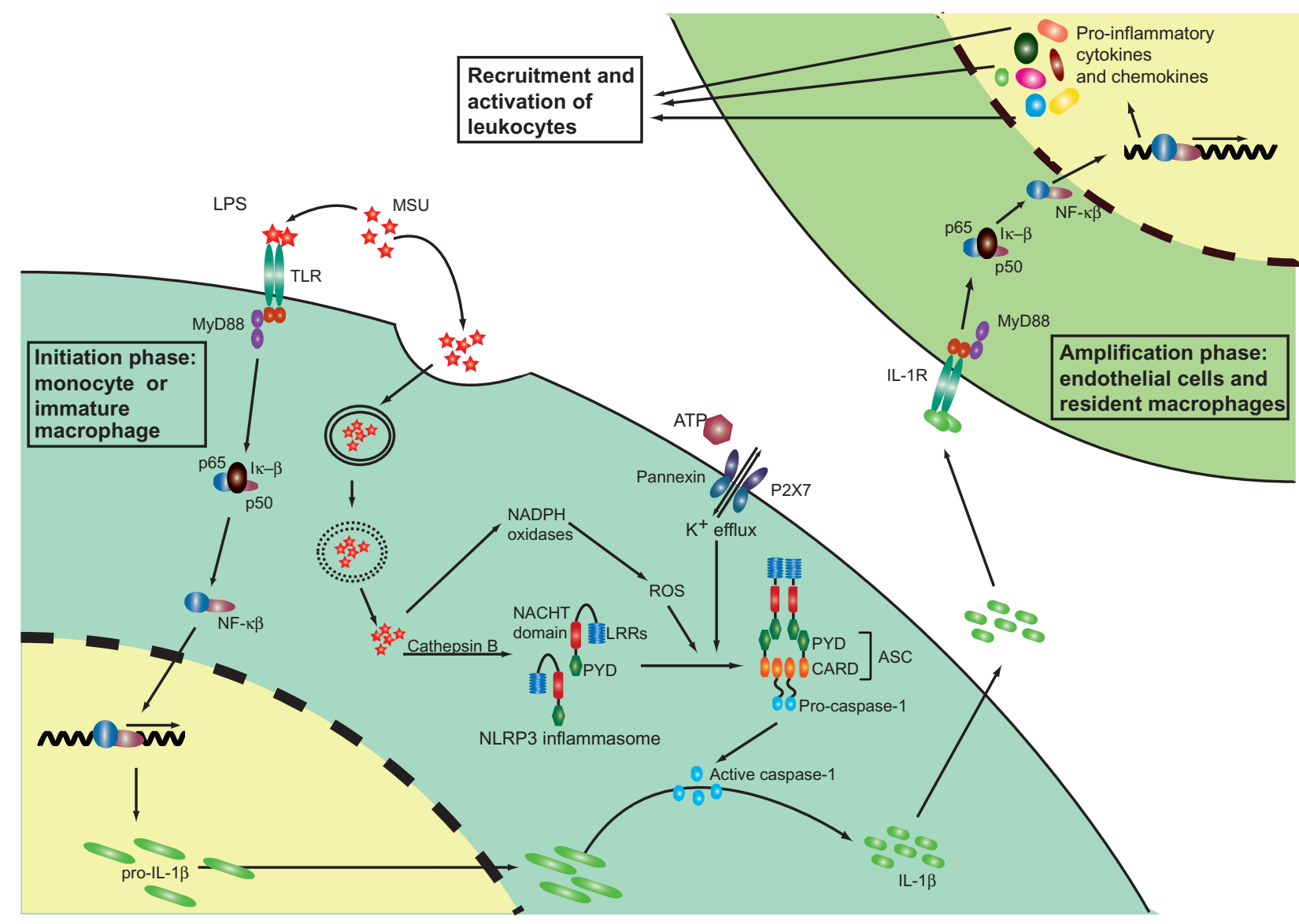

Figure 2 Inflammation in the gouty joint. Multiple steps in the inflammatory pathway are initiated by MSU deposition in the joint. MSU crystals are recognized by the pattern recognition receptors of the innate immune system, such as the TLRs and are phagocytosed by macrophages. Intracellular MSU crystals are recognized by the NLRP3 inflammasome (a multiprotein complex composed of a C-terminal LRR 'sensor' domain, a central nucleotide-binding domain (NACHT domain), which regulates selfoligomerization and an N-terminal PYD) resulting in oligomerization of NLRP3 and cleavage of procaspase-I to caspase-I. Cathepsin B, ROS, and K+ efflux (stimulated by ATP accumulation) are also understood to have some involvement in the activation and oligomerization of NLRP3 following MSU internalization. Caspase-I in turn cleaves inactive prolL- $\mid \beta$, transcribed in a NF- $\kappa \beta$-dependent manner following TLR stimulation, to produce active IL-I $\beta$, which is released into the extracellular joint fluid. IL-I $\beta$ activates ILI receptors on endothelial cells and resident macrophages within the joint, resulting in signal transduction and gene activation and leading to the secretion of an array of proinflammatory cytokines and chemokines. These in turn recruit and activate leukocytes into the joint thereby amplifying the inflammatory cascade.

Abbreviations: ASC, apoptosis-associated speck-like protein containing a CARD; CARD, caspase recruitment domain; PYD, Pyrin domain; IL, interleukin; LRR, leucinerich repeat; MSU, monosodium urate; MyD88, myeloid differentiation primary response gene (88); NACHT, domain present in neuronal apoptosis inhibitor protein (NAIP) major histocompatibility complex class II transactivator; NF- $\kappa \beta$, nuclear factor $\kappa \beta$; NLRP, NACHT domain, LRR domain, and Pyrin domain-containing protein; ROS, reactive oxygen species; TLR, Toll-like receptor.

of these diseases. Despite much progress in elucidating the biological pathways coordinating a gout attack, the precise mechanisms by which endocytosed MSU crystals activate the NLRP3 inflammasome remain unclear and it is possible that sensing may occur through intermediary protein(s). Interestingly, the reduced severity of the inflammatory response triggered by CPPD deposition in pseudogout and the inflammasome neutrality of the chemically and structurally similar allopurinol, suggests that subtle differences in the physical properties of different crystals, including surface charge and size, may be important in determining the intensity of the inflammatory response.

A recent study has shed light on the biological mechanisms underlying the association between excessive consumption of food and onset of gout with the demonstration of a synergistic relationship between free fatty acids (FFA) and MSU for the activation of IL- $1 \beta{ }^{88}$ In this study Joosten and colleagues demonstrated that MSU crystals alone were unable to induce IL- $1 \beta$ release from peripheral blood mononuclear cell (PBMC) isolated from healthy subjects; however in the presence of FFA, large amounts of active IL- $1 \beta$ were detected. Notably, IL-1 $\beta$ release in response to MSU and FFA injection was significantly reduced in caspase- 1 and ASC deficient mice, but not in NLRP3 gene deficient mice. ${ }^{88}$ How these data fit with earlier studies indicating dependency on NLRP3 for MSU-induced IL-1 $\beta$ production is unclear and may point to the involvement of alternative inflammasome complexes 
in the response to MSU or relate to differences in cell lineage, cell priming, and/or crystal structure between the studies.

In addition to the intracellular NLRP3 receptor, the extracellular TLR2 and TLR4 receptors may also play a role in the innate immune response to MSU deposition. ${ }^{89}$ Macrophages isolated from TLR ${ }^{2--/}$ and TLR $^{4-/}$ mice show impaired uptake of MSU crystals and reduced proinflammatory cytokine production, suggesting these TLRs to be essential for MSU crystal-induced inflammation ${ }^{89}$ However conflicting results from a peritoneal model of acute gout suggest TLR2 and TLR4 to be dispensable in the immune response to MSU crystals..$^{90}$ Nevertheless, as mentioned briefly above, it is conceivable that the contradictory results of these two studies may be attributable to cells in different tissues responding to MSU stimulation through different mechanisms. More recent studies now suggest that rather than having a role in the recognition of MSU crystals, the TLRs may play a more indirect part in responding to MSU deposition through regulation of the synthesis of pro-IL-1 $\beta$ (Figure 2)..$^{90,91}$

Together these studies allow a picture to be painted of our current understanding of the inflammatory process of an acute gout attack (Figure 2). During the initiation phase, MSU crystals precipitated within the joint stimulate extracellular TLR receptors expressed by resident monocytes, leading to transcription of pro-IL-1 $\beta$. MSU crystals are also phagocytosed by resident monocytes, an activity which is thought to be positively regulated by TLR activation, ${ }^{89}$ resulting in oligomerization of the NLRP3 inflammasome, activation of caspase- 1 , and subsequent cleavage of pro-IL-1 $\beta$ to produce active IL-1 $\beta$. As discussed above, $\mathrm{K}+$ efflux, ROS, and/ or cathepsin B may also be involved in mediating NLRP3 activation. Following cleavage, active IL-1 $\beta$ is released by the resident monocytes and binds to IL-1 receptors expressed by endothelial cells and resident macrophages. During the 'amplification phase' these cells respond to IL- $1 \beta$ by producing an array of proinflammatory cytokines and chemokines, which act together to coordinate the recruitment and activation of leukocytes into the joint.

One of the characteristic features of gout is that regardless of treatment, gout attacks are self-limiting. However, although great strides have been made in unraveling the molecular mechanisms of gout onset, our understanding of the mechanisms leading to resolution of a gout attack remain far less advanced. A range of possible mechanisms have been suggested, including the binding of crystals to inhibitory proteins, ${ }^{92-94}$ maturation of monocytes to macrophages, which express the anti-inflammatory TGF- $\beta 1$ in response to MSU, ${ }^{95,96}$ and clearance of apoptotic leukocytes by macrophages..$^{97,98}$ The balance between the multiple regulatory mechanisms that appear to be involved in mediating activation of the inflammasome pathway by MSU crystals, including ROS and $\mathrm{K}+$ efflux, and inhibitors of the inflammasome pathway, including caspase-12 and CD4+ memory T-cells, may also be important in the down regulation of the inflammatory response that occurs during the resolution of an inflammatory gout attack.

Gout therapy has remained very much unchanged for the last 50 years. The British Society for Rheumatology recommends nonsteroidal anti-inflammatories (NSAIDs) as the first drug of choice for an acute gout attack, followed by systemic or intra-articular glucocorticosteroids where NSAIDs are contraindicated. ${ }^{99}$ The third line of treatment is oral colchicine, which is highly effective when given early in an acute gouty attack, but is poorly tolerated because of predictable gastrointestinal side effects. Notably, colchicine has been shown to suppress MSU crystal-induced NLRP3 inflammasome protein complex assembly, most likely through microtubule inhibition and subsequent impaired delivery of MSU crystals to the NLRP3 inflammatory protein complex in the cytosol, although this effect requires relatively high (micromolar) concentrations of colchicine. ${ }^{15}$ Recent animal model studies, using anakinra (an IL-1R antagonist) and mIL-1 Trap (an IL-1 inhibitor), clearly demonstrate the potential of targeting IL- $1 \beta$ for the management of MSU-induced inflammation., ${ }^{4,5}$ Moreover, early clinical studies of the IL-1 inhibitors, rilonacept (IL-1 Trap), ${ }^{6}$ canakinumab (monoclonal anti-IL-1 $\beta$ antibody), and anakinra, ${ }^{7,8,100}$ have demonstrated efficacy in the treatment of patients with acute and chronic gout and have been generally well tolerated, although there are always concerns about increased incidence of infections when proinflammatory cytokines are inhibited and it should be noted that this has been observed in a subset of patients in a number of IL-1 inhibitor trials. These studies, coupled to in vitro evidence of the key role of the inflammasome pathway in activating IL-1 $\beta$ in gout, point to IL-1 $\beta$ and the upstream inflammasome regulatory pathways as key targets for the future treatment of gout.

\section{Conclusions and implications for future research}

Gout is the most common form of inflammatory arthritis in older men, affecting $1 \%-2 \%$ of adults in developed countries. Despite its prevalence, treatment options for gout have 
remained static for almost half a century, with the approval of allopurinol in 1965 representing the last drug approved by the US Food and Drug Administration for treating gout. However, the last year has seen the approval of a new gout therapy, the xanthine oxidase inhibitor, febuxostat, and with several new drugs now in the late stages of clinical testing ${ }^{6,9,101}$ coupled with our enhanced level of understanding of the pathophysiology of the inflammatory process involved, we are entering a new era for the treatment of gout.

Although the precise molecular details of the NLRP3 inflam masome pathway and its response to MSU crystal deposition remain to be determined, the importance of inflammasomes and IL-1 $\beta$ activation to the pathology of gout is now firmly established. Notably, current strategies for targeting IL-1 $\beta$ have proved successful for alleviating the symptoms of gout in small clinical studies suggesting that targeting this and other components of the pathway may have an important place in gout and pseudogout treatment. ${ }^{6-9}$ Moreover, the impressive results of IL-1 inhibitors for the treatment of autoinflammatory syndromes confirm the potential of these agents for diseases such as gout, which involve excessive IL- $1 \beta$ production.

Despite significant advances in our understanding of the inflammatory processes involved in an acute gout attack, many questions remain to be addressed. The precise mechanism by which crystal recognition is achieved remains elusive, whilst the role of additional cytokines, which may be activated by the NLRP3 inflammasome, such as IL-18, must also be evaluated. Moreover, MSU may trigger other pathways aside from the NLRP3 inflammasome and it will be important to identify such pathways and understand their interaction with the NLRP3 inflammasome and IL-1 $\beta$ production in the acute and resolution phases of gout. Answers to these questions will no doubt provide important insights into the mechanisms underlying MSU-induced inflammation and resolution, but may also reveal novel biologically relevant targets for the development of new, more effective therapeutic options for the treatment of gout.

\section{Acknowledgments}

SR Kingsbury and PG Conaghan are supported by the Leeds Musculoskeletal Biomedical Research Unit and Arthritis Research UK, and MF McDermott by FP7-HEALTH-20072.4.4-1 grant and Arthritis Research UK.

\section{Abbreviations}

ASC, apoptosis-associated speck-like protein containing a CARD; bZIP, basic leucine zipper domain;
CARD, caspase recruitment domain; CC, coiled-coil domain; CINCA/NOMID, chronic infantile neurologic, cutaneous, and articular syndrome/neonatal onset multisystem inflammatory disease; CPPD, calcium pyrophosphate dihydrate; DAMP, danger-associated molecular pattern; DIRA, deficiency of the interleukin-1-receptor antagonist; FCAS, familial cold autoinflammatory syndrome; FFA, free fatty acids; FIIND, domain with a function to find; FMF, familial Mediterranean fever; ICE, IL-1-converting enzyme; IL, interleukin; INCA (Inhibitory CARD); IRF, interferon-regulatory factor; LPS, lipopolysaccharide; LRR, leucine-rich repeat; $\mathrm{MSU}$, monosodium urate; MDP, muramyl dipeptide; MyD88, myeloid differentiation primary response gene (88); MWS, Muckle-Wells syndrome; NACHT, domain present in neuronal apoptosis inhibitor protein (NAIP) major histocompatibility complex class II transactivator; NF- $\kappa \beta$, nuclear factor $\kappa \beta$; NLRP, NACHT domain, LRR domain and Pyrin domain-containing protein; NLR, NOD-like receptor; PAMP, pathogen-associated molecular pattern; poly(I:C), polycytidylic acid; PAPA, pyogenic arthritis; PAPA, pyodermagangrenosum, and acne; PBMC, peripheral blood mononuclear cell; PRR, pattern-recognition receptors; PSTPIP1, proline-serine-threonine-phosphatase-interacting protein; PYD, Pyrin domain; PYPAF1, Pyrin-containing Apaf1-like protein 1; RLH, RIG-like helicase; ROS, reactive oxygen species; TLR, Toll-like receptor.

\section{Disclosure}

The authors report no conflicts of interest in this work.

\section{References}

1. Carl Wilhelm Scheele (1742-1786) Swedish apothecary. JAMA. 1970; 212(13):2258-2259.

2. Freudweiler M. Studies on the nature of gouty tophi. Dtsch Arch Klin Med. 1899;63:266.

3. Landis RC, Haskard DO. Pathogenesis of crystal-induced inflammation. Curr Rheumatol Rep. 2001;3(1):36-41.

4. Martin WJ, Walton M, Harper J. Resident macrophages initiating and driving inflammation in a monosodium urate monohydrate crystalinduced murine peritoneal model of acute gout. Arthritis Rheum. 2009; 60(1):281-289.

5. Torres R, Macdonald L, Croll SD, et al. Hyperalgesia, synovitis and multiple biomarkers of inflammation are suppressed by interleukin 1 inhibition in a novel animal model of gouty arthritis. Ann Rheum Dis. 2009;68(10):1602-1608.

6. Terkeltaub R, Sundy JS, Schumacher HR, et al. The interleukin 1 inhibitor rilonacept in treatment of chronic gouty arthritis: results of a placebocontrolled, monosequence crossover, non-randomised, single-blind pilot study. Ann Rheum Dis. 2009;68(10):1613-1617.

7. McGonagle D, Tan AL, Shankaranarayana S, Madden J, Emery P, McDermott MF. Management of treatment resistant inflammation of acute on chronic tophaceous gout with anakinra. Ann Rheum Dis. 2007;66(12):1683-1684.

8. So A, De Smedt T, Revaz S, Tschopp J. A pilot study of IL-1 inhibition by anakinra in acute gout. Arthritis Res Ther. 2007;9(2):R28. 
9. So A, De Meulemeester M, Pikhlak A, et al. Canakinumab for the treatment of acute flares in difficult-to-treat gouty arthritis: results of a multicenter, phase II, dose-ranging study. Arthritis Rheum. 2010;62(10): 3064-3076.

10. Pelegrin P, Surprenant A. Pannexin-1 couples to maitotoxin- and nigericin-induced interleukin-1beta release through a dye uptakeindependent pathway. J Biol Chem. 2007;282(4):2386-2394.

11. Maitra R, Clement CC, Scharf B, et al. Endosomal damage and TLR2 mediated inflammasome activation by alkane particles in the generation of aseptic osteolysis. Mol Immunol. 2009;47(2-3):175-184.

12. Hornung V, Bauernfeind F, Halle A, et al. Silica crystals and aluminum salts activate the NALP3 inflammasome through phagosomal destabilization. Nat Immunol. 2008;9(8):847-856.

13. Hacker H, Vabulas RM, Takeuchi O, Hoshino K, Akira S, Wagner H. Immune cell activation by bacterial CpG-DNA through myeloid differentiation marker 88 and tumor necrosis factor receptor-associated factor (TRAF)6. J Exp Med. 2000;192(4):595-600.

14. Mariathasan S, Weiss DS, Newton K, et al. Cryopyrin activates the inflammasome in response to toxins and ATP. Nature. 2006;440(7081): 228-232.

15. Martinon F, Petrilli V, Mayor A, Tardivel A, Tschopp J. Gout-associated uric acid crystals activate the NALP3 inflammasome. Nature. 2006; 440(7081):237-241.

16. Zhang FX, Kirschning CJ, Mancinelli R, et al. Bacterial lipopolysaccharide activates nuclear factor-kappaB through interleukin-1 signaling mediators in cultured human dermal endothelial cells and mononuclear phagocytes. J Biol Chem. 1999;274(12):7611-7614.

17. Schwandner R, Dziarski R, Wesche H, Rothe M, Kirschning CJ. Peptidoglycan- and lipoteichoic acid-induced cell activation is mediated by toll-like receptor 2. J Biol Chem. 1999;274(25):17406-17409.

18. Moors MA, Li L, Mizel SB. Activation of interleukin-1 receptorassociated kinase by gram-negative flagellin. Infect Immun. 2001;69(7): 4424-4429.

19. Frendeus B, Wachtler C, Hedlund M, et al. Escherichia coli P fimbriae utilize the Toll-like receptor 4 pathway for cell activation. $\mathrm{Mol}$ Microbiol. 2001;40(1):37-51.

20. Hedlund M, Frendeus B, Wachtler C, Hang L, Fischer H, Svanborg C. Type 1 fimbriae deliver an LPS- and TLR4-dependent activation signal to CD14-negative cells. Mol Microbiol. 2001;39(3):542-552.

21. Nociari M, Ocheretina O, Schoggins JW, Falck-Pedersen E. Sensing infection by adenovirus: Toll-like receptor-independent viral DNA recognition signals activation of the interferon regulatory factor 3 master regulator. J Virol. 2007;81(8):4145-4157.

22. Lin Y, Lee H, Berg AH, Lisanti MP, Shapiro L, Scherer PE. The lipopolysaccharide-activated toll-like receptor (TLR)-4 induces synthesis of the closely related receptor TLR-2 in adipocytes. J Biol Chem. 2000;275(32):24255-24263.

23. Kawai T, Akira S. Antiviral signaling through pattern recognition receptors. J Biochem. 2007;141(2):137-145.

24. Martinon F, Agostini L, Meylan E, Tschopp J. Identification of bacterial muramyl dipeptide as activator of the NALP3/cryopyrin inflammasome. Curr Biol. 2004;14(21):1929-1934.

25. Craven RR, Gao X, Allen IC, et al. Staphylococcus aureus alphahemolysin activates the NLRP3-inflammasome in human and mouse monocytic cells. PLoS One. 2009;4(10):e7446.

26. Kanneganti TD, Ozoren N, Body-Malapel M, et al. Bacterial RNA and small antiviral compounds activate caspase-1 through cryopyrin/Nalp3. Nature. 2006;440(7081):233-236.

27. Muruve DA, Petrilli V, Zaiss AK, et al. The inflammasome recognizes cytosolic microbial and host DNA and triggers an innate immune response. Nature. 2008;452(7183):103-107.

28. Eisenbarth SC, Colegio OR, O'Connor W, Sutterwala FS, Flavell RA. Crucial role for the Nalp3 inflammasome in the immunostimulatory properties of aluminium adjuvants. Nature. 2008;453(7198):1122-1126.

29. Cassel SL, Eisenbarth SC, Iyer SS, et al. The Nalp3 inflammasome is essential for the development of silicosis. Proc Natl Acad Sci USA. 2008;105(26):9035-9040.
30. Dostert C, Petrilli V, Van Bruggen R, Steele C, Mossman BT, Tschopp J. Innate immune activation through Nalp3 inflammasome sensing of asbestos and silica. Science. 2008;320(5876):674-677.

31. Inohara N, Chamaillard M, McDonald C, Nunez G. NOD-LRR proteins: role in host-microbial interactions and inflammatory disease. Annu Rev Biochem. 2005;74:355-383.

32. Martinon F, Burns K, Tschopp J. The inflammasome: a molecular platform triggering activation of inflammatory caspases and processing of proIL-beta. Mol Cell. 2002;10(2):417-426.

33. Martinon F, Tschopp J. Inflammatory caspases and inflammasomes: master switches of inflammation. Cell Death Differ. 2007;14(1): $10-22$

34. Petrilli V, Papin S, Dostert C, Mayor A, Martinon F, Tschopp J. Activation of the NALP3 inflammasome is triggered by low intracellular potassium concentration. Cell Death Differ. 2007;14(9): 1583-1589.

35. Kahlenberg JM, Dubyak GR. Mechanisms of caspase-1 activation by P2X7 receptor-mediated K+ release. Am J Physiol Cell Physiol. 2004; 286(5):C1100-C1108.

36. Franchi L, Kanneganti TD, Dubyak GR, Nunez G. Differential requirement of $\mathrm{P} 2 \mathrm{X} 7$ receptor and intracellular $\mathrm{K}+$ for caspase-1 activation induced by intracellular and extracellular bacteria. J Biol Chem. 2007; 282(26):18810-18818.

37. Halle A, Hornung V, Petzold GC, et al. The NALP3 inflammasome is involved in the innate immune response to amyloid-beta. Nat Immunol. 2008;9(8):857-865.

38. Chu SC, Yang SF, Tzang BS, Hsieh YS, Lue KH, Lu KH. Cathepsin B and cystatin $\mathrm{C}$ play an inflammatory role in gouty arthritis of the knee. Clin Chim Acta. 2010;411(21-22):1788-1792.

39. Meissner F, Molawi K, Zychlinsky A. Superoxide dismutase 1 regulates caspase-1 and endotoxic shock. Nat Immunol. 2008;9(8):866-872.

40. Netea MG, Nold-Petry CA, Nold MF, et al. Differential requirement for the activation of the inflammasome for processing and release of IL-1beta in monocytes and macrophages. Blood. 2009;113(10):2324-2335.

41. Roberts TL, Idris A, Dunn JA, et al. HIN-200 proteins regulate caspase activation in response to foreign cytoplasmic DNA. Science. 2009; 323(5917):1057-1060.

42. Fernandes-Alnemri T, Yu JW, Datta P, Wu J, Alnemri ES. AIM2 activates the inflammasome and cell death in response to cytoplasmic DNA. Nature. 2009;458(7237):509-513.

43. Hornung V, Ablasser A, Charrel-Dennis M, et al. AIM2 recognizes cytosolic dsDNA and forms a caspase-1-activating inflammasome with ASC. Nature. 2009;458(7237):514-518.

44. Burckstummer T, Baumann C, Bluml S, et al. An orthogonal proteomicgenomic screen identifies AIM2 as a cytoplasmic DNA sensor for the inflammasome. Nat Immunol. 2009;10(3):266-272.

45. Humke EW, Shriver SK, Starovasnik MA, Fairbrother WJ, Dixit VM. ICEBERG: a novel inhibitor of interleukin-1beta generation. Cell. 2000; 103(1):99-111.

46. Druilhe A, Srinivasula SM, Razmara M, Ahmad M, Alnemri ES. Regulation of IL-1beta generation by Pseudo-ICE and ICEBERG, two dominant negative caspase recruitment domain proteins. Cell Death Differ. 2001;8(6):649-657.

47. Lamkanfi M, Denecker G, Kalai M, et al. INCA, a novel human caspase recruitment domain protein that inhibits interleukin-1beta generation. J Biol Chem. 2004;279(50):51729-51738.

48. Scott AM, Saleh M. The inflammatory caspases: guardians against infections and sepsis. Cell Death Differ. 2007;14(1):23-31.

49. Saleh M, Mathison JC, Wolinski MK, et al. Enhanced bacterial clearance and sepsis resistance in caspase-12-deficient mice. Nature. 2006; 440(7087):1064-1068.

50. Saleh M, Vaillancourt JP, Graham RK, et al. Differential modulation of endotoxin responsiveness by human caspase-12 polymorphisms. Nature. 2004;429(6987):75-79.

51. Guarda G, Dostert C, Staehli F, et al. T cells dampen innate immune responses through inhibition of NLRP1 and NLRP3 inflammasomes. Nature. 2009;460(7252):269-273. 
52. Hoffman HM, Mueller JL, Broide DH, Wanderer AA, Kolodner RD. Mutation of a new gene encoding a putative pyrin-like protein causes familial cold autoinflammatory syndrome and Muckle-Wells syndrome. Nat Genet. 2001;29(3):301-305.

53. Feldmann J, Prieur AM, Quartier P, et al. Chronic infantile neurological cutaneous and articular syndrome is caused by mutations in CIAS1, a gene highly expressed in polymorphonuclear cells and chondrocytes. Am J Hum Genet. 2002;71(1):198-203.

54. Aganna E, Martinon F, Hawkins PN, et al. Association of mutations in the NALP3/CIAS1/PYPAF1 gene with a broad phenotype including recurrent fever, cold sensitivity, sensorineural deafness, and AA amyloidosis. Arthritis Rheum. 2002;46(9):2445-2452.

55. Agostini L, Martinon F, Burns K, McDermott MF, Hawkins PN, Tschopp J. NALP3 forms an IL-1beta-processing inflammasome with increased activity in Muckle-Wells autoinflammatory disorder. Immunity. 2004;20(3):319-325.

56. Aksentijevich I, Nowak M, Mallah M, et al. De novo CIAS1 mutations, cytokine activation, and evidence for genetic heterogeneity in patients with neonatal-onset multisystem inflammatory disease (NOMID): a new member of the expanding family of pyrin-associated autoinflammatory diseases. Arthritis Rheum. 2002;46(12):3340-3348.

57. Brydges SD, Mueller JL, McGeough MD, et al. Inflammasomemediated disease animal models reveal roles for innate but not adaptive immunity. Immunity. 2009;30(6):875-887.

58. Hoffman HM, Firestein GS. Anakinra for the treatment of neonatalonset multisystem inflammatory disease. Nat Clin Pract Rheumatol. 2006;2(12):646-647.

59. Goldbach-Mansky R, Dailey NJ, Canna SW, et al. Neonatal-onset multisystem inflammatory disease responsive to interleukin-1beta inhibition. N Engl J Med. 2006;355(6):581-592.

60. Hawkins PN, Lachmann HJ, McDermott MF. Interleukin-1-receptor antagonist in the Muckle-Wells syndrome. N Engl J Med. 2003;348(25): 2583-2584.

61. Goldbach-Mansky R, Shroff SD, Wilson M, et al. A pilot study to evaluate the safety and efficacy of the long-acting interleukin-1 inhibitor rilonacept (interleukin-1 Trap) in patients with familial cold autoinflammatory syndrome. Arthritis Rheum. 2008;58(8):2432-2442.

62. Hoffman HM, Throne ML, Amar NJ, et al. Efficacy and safety of rilonacept (interleukin-1 Trap) in patients with cryopyrin-associated periodic syndromes: results from two sequential placebo-controlled studies. Arthritis Rheum. 2008;58(8):2443-2452.

63. Lachmann HJ, Kone-Paut I, Kuemmerle-Deschner JB, et al. Use of canakinumab in the cryopyrin-associated periodic syndrome. $N$ Engl J Med. 2009;360(23):2416-2425.

64. Lachmann HJ, Lowe P, Felix SD, et al. In vivo regulation of interleukin 1 beta in patients with cryopyrin-associated periodic syndromes. $J$ Exp Med. 2009;206(5):1029-1036.

65. Hoffman HM. Rilonacept for the treatment of cryopyrin-associated periodic syndromes (CAPS). Expert Opin Biol Ther. 2009;9(4):519-531.

66. Stack JH, Beaumont K, Larsen PD, et al. IL-converting enzyme/ caspase-1 inhibitor VX-765 blocks the hypersensitive response to an inflammatory stimulus in monocytes from familial cold autoinflammatory syndrome patients. J Immunol. 2005;175(4):2630-2634.

67. Masters SL, Simon A, Aksentijevich I, Kastner DL. Horror autoinflammaticus: the molecular pathophysiology of autoinflammatory disease. Annu Rev Immunol. 2009;27:621-668.

68. Wise CA, Gillum JD, Seidman CE, et al. Mutations in CD2BP1 disrupt binding to PTP PEST and are responsible for PAPA syndrome, an autoinflammatory disorder. Hum Mol Genet. 2002;11(8):961-969.

69. Shoham NG, Centola M, Mansfield E, et al. Pyrin binds the PSTPIP1/ CD2BP1 protein, defining familial Mediterranean fever and PAPA syndrome as disorders in the same pathway. Proc Natl Acad Sci U SA. 2003;100(23):13501-13506.

70. Verma D, Lerm M, Blomgran Julinder R, Eriksson P, Soderkvist P, Sarndahl E. Gene polymorphisms in the NALP3 inflammasome are associated with interleukin-1 production and severe inflammation: relation to common inflammatory diseases? Arthritis Rheum. 2008;58(3):888-894.
71. Kastbom A, Verma D, Eriksson P, Skogh T, Wingren G, Soderkvist P. Genetic variation in proteins of the cryopyrin inflammasome influences susceptibility and severity of rheumatoid arthritis (the Swedish TIRA project). Rheumatology (Oxford). 2008;47(4):415-417.

72. Schoultz I, Verma D, Halfvarsson J, et al. Combined polymorphisms in genes encoding the inflammasome components NALP3 and CARD8 confer susceptibility to Crohn's disease in Swedish men. Am J Gastroenterol. 2009;104(5):1180-1188.

73. Miao ZM, Zhao SH, Yan SL, et al. NALP3 inflammasome functional polymorphisms and gout susceptibility. Cell Cycle. 2009;8(1):27-30.

74. Aksentijevich I, Masters SL, Ferguson PJ, et al. An autoinflammatory disease with deficiency of the interleukin-1-receptor antagonist. $N E n g l$ J Med. 2009;360(23):2426-2437.

75. Masters SL, Dunne A, Subramanian SL, et al. Activation of the NLRP3 inflammasome by islet amyloid polypeptide provides a mechanism for enhanced IL-1beta in type 2 diabetes. Nat Immunol. 2010; 11(10):897-904.

76. Duewell P, Kono H, Rayner KJ, et al. NLRP3 inflammasomes are required for atherogenesis and activated by cholesterol crystals. Nature. 2010;464(7293):1357-1361.

77. Rajamaki K, Lappalainen J, Oorni K, et al. Cholesterol crystals activate the NLRP 3 inflammasome in human macrophages: a novel link between cholesterol metabolism and inflammation. PLoS One. 2010;5(7): e11765.

78. Okamoto M, Liu W, Luo Y, et al. Constitutively active inflammasome in human melanoma cells mediating autoinflammation via caspase-1 processing and secretion of interleukin-1beta. J Biol Chem. 2010;285(9): 6477-6488.

79. Vilaysane A, Chun J, Seamone ME, et al. The NLRP3 inflammasome promotes renal inflammation and contributes to CKD. JAm Soc Nephrol. 2010;21(10):1732-1744.

80. Kanneganti TD, Body-Malapel M, Amer A, et al. Critical role for Cryopyrin/Nalp3 in activation of caspase-1 in response to viral infection and double-stranded RNA. J Biol Chem. 2006;281(48):36560-36568.

81. Allen IC, Scull MA, Moore CB, et al. The NLRP3 inflammasome mediates in vivo innate immunity to influenza A virus through recognition of viral RNA. Immunity. 2009;30(4):556-565.

82. Ichinohe T, Lee HK, Ogura Y, Flavell R, Iwasaki A. Inflammasome recognition of influenza virus is essential for adaptive immune responses. J Exp Med. 2009;206(1):79-87.

83. Kanneganti TD. Central roles of NLRs and inflammasomes in viral infection. Nat Rev Immunol. 2010;10(10):688-698.

84. Allen IC, TeKippe EM, Woodford RM, et al. The NLRP3 inflammasome functions as a negative regulator of tumorigenesis during colitis-associated cancer. J Exp Med. 2010;207(5):1045-1056.

85. Yagnik DR, Hillyer P, Marshall D, et al. Noninflammatory phagocytosis of monosodium urate monohydrate crystals by mouse macrophages. Implications for the control of joint inflammation in gout. Arthritis Rheum. 2000;43(8):1779-1789.

86. Landis RC, Yagnik DR, Florey O, et al. Safe disposal of inflammatory monosodium urate monohydrate crystals by differentiated macrophages. Arthritis Rheum. 2002;46(11):3026-3033.

87. Shi Y, Evans JE, Rock KL. Molecular identification of a danger signal that alerts the immune system to dying cells. Nature. 2003;425(6957): 516-521.

88. Joosten LA, Netea MG, Mylona E, et al. Engagement of fatty acids with Toll-like receptor 2 drives interleukin-1beta production via the ASC/ caspase 1 pathway in monosodium urate monohydrate crystal-induced gouty arthritis. Arthritis Rheum. 2010;62(11):3237-3248.

89. Liu-Bryan R, Scott P, Sydlaske A, Rose DM, Terkeltaub R. Innate immunity conferred by Toll-like receptors 2 and 4 and myeloid differentiation factor 88 expression is pivotal to monosodium urate monohydrate crystal-induced inflammation. Arthritis Rheum. 2005;52(9): 2936-2946.

90. Chen CJ, Shi Y, Hearn A, et al. MyD88-dependent IL-1 receptor signaling is essential for gouty inflammation stimulated by monosodium urate crystals. J Clin Invest. 2006;116(8):2262-2271. 
91. Scott P, Ma H, Viriyakosol S, Terkeltaub R, Liu-Bryan R. Engagement of CD14 mediates the inflammatory potential of monosodium urate crystals. J Immunol. 2006;177(9):6370-6378.

92. Terkeltaub R, Curtiss LK, Tenner AJ, Ginsberg MH. Lipoproteins containing apoprotein $\mathrm{B}$ are a major regulator of neutrophil responses to monosodium urate crystals. J Clin Invest. 1984;73(6):1719-1730.

93. Terkeltaub RA, Dyer CA, Martin J, Curtiss LK. Apolipoprotein (apo) E inhibits the capacity of monosodium urate crystals to stimulate neutrophils. Characterization of intraarticular apo $\mathrm{E}$ and demonstration of apo E binding to urate crystals in vivo. J Clin Invest. 1991;87(1): 20-26.

94. Ortiz-Bravo E, Sieck MS, Schumacher HR Jr. Changes in the proteins coating monosodium urate crystals during active and subsiding inflammation. Immunogold studies of synovial fluid from patients with gout and of fluid obtained using the rat subcutaneous air pouch model. Arthritis Rheum. 1993;36(9):1274-1285.

95. Liote F, Prudhommeaux F, Schiltz C, et al. Inhibition and prevention of monosodium urate monohydrate crystal-induced acute inflammation in vivo by transforming growth factor beta1. Arthritis Rheum. 1996; 39(7):1192-1198.

96. Redini F, Mauviel A, Pronost S, Loyau G, Pujol JP. Transforming growth factor beta exerts opposite effects from interleukin-1 beta on cultured rabbit articular chondrocytes through reduction of interleukin-1 receptor expression. Arthritis Rheum. 1993;36(1):44-50.
97. Fadok VA, Bratton DL, Konowal A, Freed PW, Westcott JY, Henson PM. Macrophages that have ingested apoptotic cells in vitro inhibit proinflammatory cytokine production through autocrine/ paracrine mechanisms involving TGF-beta, PGE2, and PAF. $J$ Clin Invest. 1998;101(4):890-898.

98. Bellingan GJ, Caldwell H, Howie SE, Dransfield I, Haslett C. In vivo fate of the inflammatory macrophage during the resolution of inflammation: inflammatory macrophages do not die locally, but emigrate to the draining lymph nodes. J Immunol. 1996;157(6):2577-2585.

99. Jordan KM, Cameron JS, Snaith M, et al. British Society for Rheumatology and British Health Professionals in Rheumatology guideline for the management of gout. Rheumatology (Oxford). 2007;46(8): 1372-1374.

100. McGonagle D, Tan AL, Madden J, Emery P, McDermott MF. Successful treatment of resistant pseudogout with anakinra. Arthritis Rheum. 2008;58(2):631-633.

101. Lasko B, Sheedy B, Hingorani V. RDEA594, a novel uricosuric agent, significantly reduced serum urate levels and was well tolerated in a phase 2a pilot study in hyperuricemic gout patients. Arthritis Rheum. 2009;60:S413-S414.
Journal of Inflammation Research

\section{Publish your work in this journal}

The Journal of Inflammation Research is an international, peer-reviewed open-access journal that welcomes laboratory and clinical findings on the molecular basis, cell biology and pharmacology of inflammation including original research, reviews, symposium reports, hypothesis formation and commentaries on: acute/chronic inflammation; mediators of inflamma-

\section{Dovepress}

tion; cellular processes; molecular mechanisms; pharmacology and novel anti-inflammatory drugs; clinical conditions involving inflammation. The manuscript management system is completely online and includes a very quick and fair peer-review system. Visit http://www.dovepress.com/ testimonials.php to read real quotes from published authors. 